Gynecologic and

Obstetric Investigation
Gynecol Obstet Invest 2018;83:417-427

DOI: $10.1159 / 000482008$
Received: June 2, 2017

Accepted: October 5, 2017

Published online: April 12, 2018

\title{
The Risk of Human Papillomavirus Infection for Spontaneous Abortion, Spontaneous Preterm Birth, and Pregnancy Rate of Assisted Reproductive Technologies: A Systematic Review and Meta-Analysis
}

\author{
Yi-Quan Xiong ${ }^{\mathrm{a}}$ Yun Mo ${ }^{\mathrm{a}}$ Qiao-Ming Luo ${ }^{\mathrm{b}}$ Shu-Ting Huo ${ }^{\mathrm{a}}$ Wen-Qiao He \\ Qing Chen ${ }^{\text {a }}$ \\ ${ }^{a}$ Department of Epidemiology, School of Public Health, Guangdong Provincial Key Laboratory of Tropical \\ Disease Research, Southern Medical University, Guangzhou, China; ${ }^{b}$ Preventive Medicine (Health Inspection and \\ Quarantine), School of Public Health, Southern Medical University, Guangzhou, China
}

\section{Keywords}

Human papillomavirus · Spontaneous abortion .

Spontaneous preterm birth - Assisted reproductive

technologies $\cdot$ Meta-analysis

\begin{abstract}
Aims: To estimate the impact of human papillomavirus (HPV) infection on spontaneous abortion, spontaneous preterm birth (sPTB), pregnancy rate of females undergoing assisted reproductive technologies (ART), and spontaneous abortion of ART pregnancy. Methods: PubMed, Medline, Embase, and the Cochrane Library were searched until December 16, 2016. The OR or relative risk (RR) with its corresponding $95 \%$ $\mathrm{Cl}$ were selected as effect size. Subgroup analysis of HPV genotype infection (high-risk HPV [HR-HPV] or indiscriminate genotype) was performed. Statistical analysis was conducted using STATA 12.0. Results: Eighteen studies were included. Eight studies revealed no significant association between HPV infection and spontaneous abortion (OR 1.40, 95\% Cl 0.56-3.50). However, subgroup analysis showed indiscriminate genotype HPV infection increased the ratio of sponta-
\end{abstract}

neous abortion with OR of $2.24(95 \% \mathrm{Cl} 1.37-3.65)$, while HRHPV infection had no significant effect (OR $0.65,95 \% \mathrm{Cl} 0.21-$ 1.98). The results indicated that HR-HPV infection was a risk for sPTB with a pooled OR of 2.84 (95\% Cl 1.95-4.14). HPV infection was found to be independent of the ART-based clinical pregnancy rate (RR 1.04, 95\% Cl 0.64-1.70) and spontaneous abortion of ART pregnancy (RR 1.47, 95\% Cl 0.862.50). Conclusions: Indiscriminate HPV genotype infection can increase the risk of spontaneous abortion and HR-HPV infection was a risk factor for SPTB. However, there was not enough evidence to indicate the association between HPV infection and pregnancy rate of ART, and spontaneous abortion of ART pregnancy. Different genotypes of HPV infection may play a discrepant role in adverse pregnancy outcomes.

(c) 2018 S. Karger AG, Basel

\section{Introduction}

Human papillomavirus (HPV) is one of the most prevalent sexually transmitted viral infections in men and women worldwide [1]. The existence of more than 180

\section{KARGER}

(c) 2018 S. Karger AG, Basel

E-Mail karger@karger.com

www.karger.com/goi 
HPV genotypes has been reported [2]. According to the basis of oncogenic potential, HPV can be divided into 2 different groups-high-risk HPV (HR-HPV) and low-risk HPV (LR-HPV). Generally, the HR-HPV included HVP16, HPV18, HPV31, HPV33, HPV35, HPV39, HPV45, HPV51, HPV52, HPV56, HPV58, HPV59, HPV66, and HPV68 [3]. It is well established that infections with HR-HPV can lead to cervical cancer. Moreover, recent evidence has shown that HPV infection is associated with the risk of colorectal, head, and oropharyngeal carcinomas $[4,5]$.

To date, a succession of studies has been published to show the association between HPV infection and adverse pregnancy outcomes. A study reported that HPV was positive in $34(24.4 \%)$ of 139 cervical samples from women who experienced spontaneous abortion. Additionally, the authors reported that $27.3 \%$ of HPV-positive women were found to have repetitive abortions, whereas this rate was only $17.4 \%$ in HPV-negative women [6]. A systematic review showed that the prevalence of HPV was significantly higher in the cervix of women who had spontaneous abortions than those who had normal full-term pregnancies ( 24.5 vs. $17.5 \%)$; this trend demonstrated a probable association between spontaneous abortion and the infection of HPV [7].

Recent evidence suggests that HPV infection may affect fertility and the clinical pregnancy rate of assisted reproductive technologies (ART). For instance, HPV infection can affect the parameters of sperm quality, and may play a crucial role in male fertility $[8,9]$. However, there are controversial results whether HPV infection may reduce the clinical pregnancy and live birth rates in women undergoing ART [10-12]. Spandorfer et al. [10] reported that HPV-positive women had a decreased pregnancy rate ( 4 of $17,23.5 \%$ ) as compared with HPVnegative women (51 of $89,57.3 \%$; $p<0.02)$. Spandorfer et al. [10] reported that patients with HPV infection were less likely to become pregnant after undergoing in vitro fertilization (IVF), and clinical pregnancy rate was lower in the group of patients with HPV positive (HPV positive vs. negative: 23.5 vs. $51.7 \%$ ). However, a prospective study including 362 patients indicated no significant difference in pregnancy rates between HPVpositive and HPV-negative women undergoing IVF treatment [12].

Considering the relationship of HPV infection and spontaneous abortion, spontaneous preterm birth (sPTB) and ART outcomes could have far-reaching implications in the medical care of pregnant and infertile women. Thus, we conducted this meta-analysis with a summary of all published estimates to evaluate the effect of HPV infection in spontaneous abortion, sPTB, and clinical pregnancy rates of females undergoing ART.

\section{Methods}

This meta-analysis is conducted in accordance with the Preferred Reporting Items for Systematic Reviews and Meta-Analyses guidelines [13].

\section{Literature Search}

We searched the PubMed, Medline, Embase, and Cochrane Library database from the earliest date available up until December 16, 2016. We used the following keywords separately and in combinations: "human papillomavirus," "HPV," "miscarriage," "abortion," "pregnancy," "preterm birth," "PROM," "premature rupture of the membranes," "in vitro fertilization," "IVF," "assisted reproductive technology," "ART," and "ICSI." Manual searches of the reference lists of review articles and studies included in the final publication selection were also conducted.

\section{Inclusion and Exclusion Criteria}

Studies were screened for eligibility if they met the following criteria (1) reported spontaneous abortion, sPTB, and ART outcomes in patients with HPV infection; (2) reported corresponding effect estimates or sufficient data for their calculation; (3) cohort study, case-control study, or cross-sectional study design; and (4) HPV DNA in cervical or placental samples was detected to diagnose the infection by polymerase chain reaction or other methods. Studies were excluded for the following reasons: (1) if they were review articles, editorials, opinions, or case reports; (2) if they did not include effective control groups to calculate effect estimates; and (3) if cervical cytology was used as a surrogate for HPV infection.

\section{Data Selection and Extraction}

Citations were merged using Endnote (version X7) to facilitate management and data extraction. Two authors independently evaluated all retrieved articles by title, abstract, and full text according to the above inclusion criteria. Any disagreement was resolved by consensus. Data were independently extracted from each eligible study and included first author, publication year, country of origin, study design, HPV detection method, HPV genotype, number of HPV infected participants with or without spontaneous abortion, sPTB and pregnancy after ART, corresponding relative risk (RRs) or ORs, and 95\% CI values. When data were reported from overlapping study samples (e.g., multiple publications from the same study), the most recent and comprehensive report was considered.

\section{Quality Assessment}

The quality of case control and cohort studies was assessed by using the Newcastle-Ottawa Scale $[14,15]$. In this scale, studies are scored across 3 categories: selection of subjects, comparability of study groups, and assessment of outcome/exposure. 
Fig. 1. Flow diagram of the studies identified in the meta-analysis.

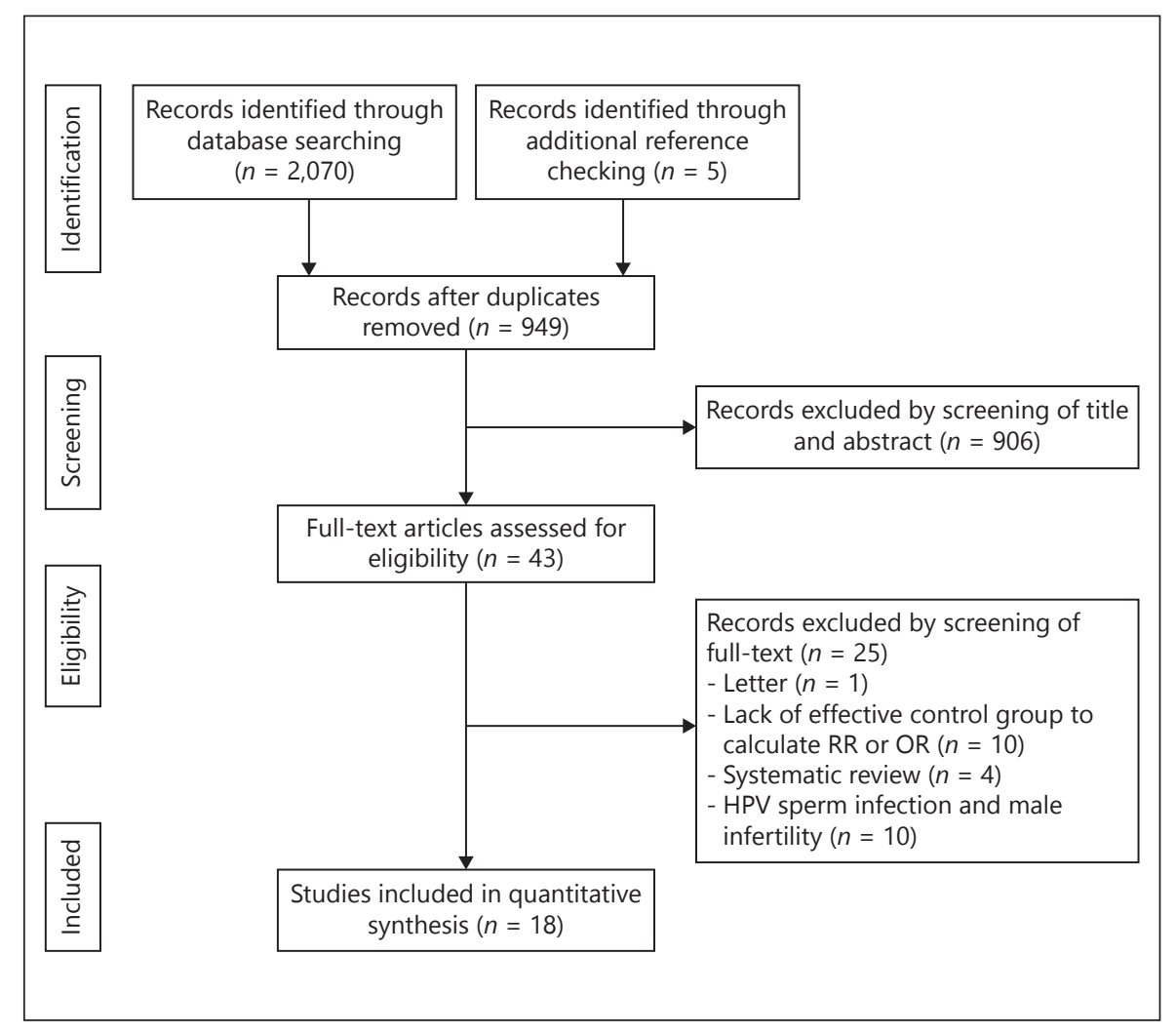

The rating system was used to indicate the quality of a study, with a maximum score of 9 . Studies were graded on an ordinal scoring scale, with higher scores representing studies of higher quality.

\section{Statistical Analysis}

The ORs or RRs with their corresponding 95\% CIs were selected as the effect size to assess the risk of spontaneous abortion, sPTB, and pregnancy after ART in HPV-infected subjects. Cohort studies and case-control studies were included in the pooled results, while cross-sectional studies were included only in the sensitivity analysis. Inter-study heterogeneity was estimated by the $I^{2}$ statistic, and significant heterogeneity was defined as $I^{2}$ $\geq 50 \%$. Pooled results and corresponding 95\% CIs were calculated with a fixed effects model (Mantel and Haenszel method) when heterogeneity was not significant $\left(I^{2}<50 \%\right)$; otherwise, a random-effects model (DerSimonian and Laird method) was applied. Forest plots were constructed for visual display of pooled results if necessary. Subgroup analyses were performed according to study design (cohort study or case-control study) and HPV genotype infection pattern (HR-HPV alone or indiscriminate genotype [HR/LR-HPV]). A meta-regression analysis was also performed to explore inter-study heterogeneity. Moreover, sensitivity analyses were used to evaluate whether any single study dominated the results of the meta-analyses. Finally, publication bias was assessed by the visual inspection of funnel plots and Egger's linear regression [16]. Statistical analyses were conducted using STATA 12.0 (Stata Corp LP, College Station, TX, USA).

HPV Infection and Pregnancy Outcomes

\section{Results}

\section{Description of Included Studies}

The systematic literature searches identified 2070 potentially relevant studies. Most ineligible studies were excluded based on the information in the title or abstract. The selection process is shown in Figure 1. Eighteen studies [10-12, 17-31] were included in our analysis. The main characteristics of included studies are described in Table 1. Twelve studies [12, 19-22, 24-30] reported the impact of HPV infection on risk of spontaneous abortion. Five studies $[17,18,21,23,31]$ reported the risk of sPTB and 7 studies $[10-12,19-22]$ reported the risk of clinical pregnancy rate of ART. Eight studies [12, 17, 18, 20, 21, $23,27,29]$ reported HR-HPV infection alone and the other 10 studies [10, 11, 19, 22, 24-26, 28, 30, 31] reported indiscriminate genotype infection (HR/LR-HPV).

\section{Association between HPV Infection and Spontaneous Abortion}

Twelve studies $[12,19-22,24-30]$ including 5 cohort studies, 3 case-control studies, and 4 cross-sectional studies, with a total of 3,007 participants, reported the impact of HPV infection on spontaneous abortion. Pooled results 


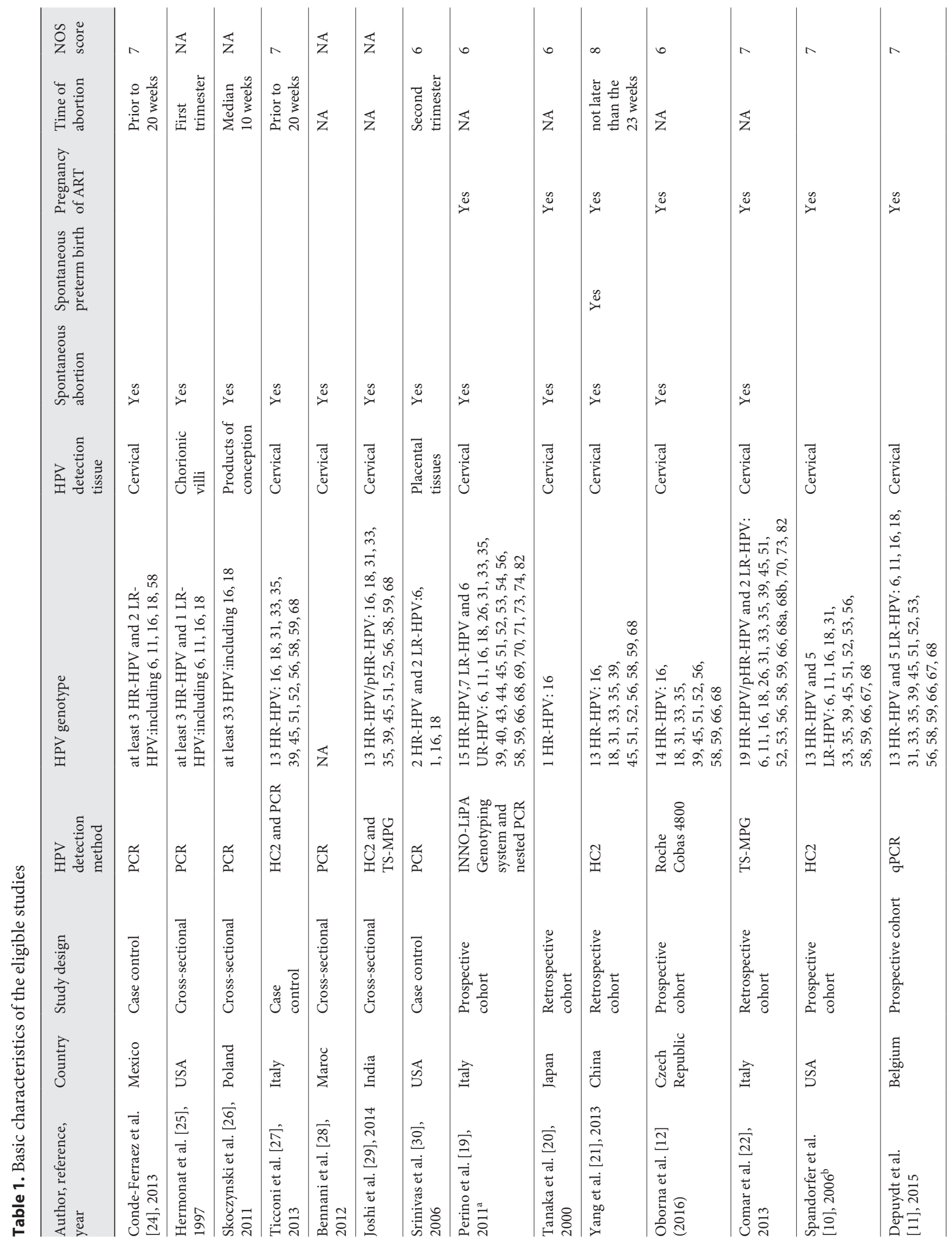




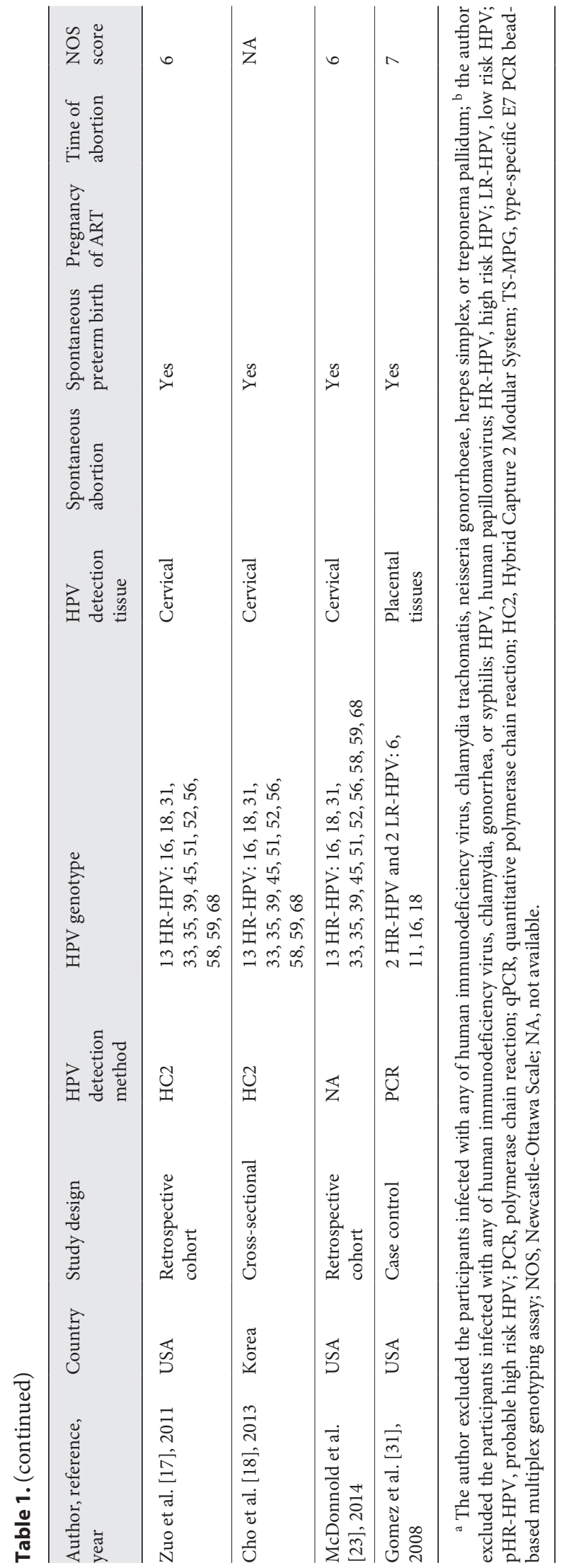

HPV Infection and Pregnancy Outcomes from 8 cohort/case-control studies suggested that infection with HPV probably increased the ratio of spontaneous abortion by 1.40 ( $95 \%$ CI $0.56-3.50 ; I^{2}=79.4 \%$; Fig. 2$)$. The pooled ORs of cohort $[12,19-22]$ and case-control studies $[24,27,30]$ were $1.47\left(95 \% \mathrm{CI} 0.86-2.50 ; I^{2}=8.3 \%\right)$ and 1.02 $\left(95 \%\right.$ CI $\left.0.21-5.02 ; I^{2}=92.4 \%\right)$ respectively (Table 2$)$. When only HR-HPV infection was considered, the pooled OR from 3 cohort studies and one case-control study involving a total of 456 participants was 0.65 (95\% CI $0.21-1.98, I^{2}=$ 64.7\%). The results of HR-HPV infection alone and all HPV genotypes' infection indicated that there was no significant association between HPV infection and spontaneous abortion. However, when removed from the HR-HPV infection studies, the pooled results based on 4 cohort/case control studies indicated that indiscriminate genotype HPV infection increased the ratio of spontaneous abortion with OR of 2.24 (95\% CI 1.37-3.65; $I^{2}=0.0 \%$; Table 2).

\section{Association between HPV Infection and SPTB}

Five studies [12, 17, 18, 21, 23], including 3 cohort studies, one case-control study, and one cross-sectional study, with a total of 1,719 participants, reported the impact of cervical HPV infection on the risk of sPTB. All 5 studies studied HR-HPV infection. The pooled results of cohort/case-control studies indicated that HR-HPV infection was a risk factor for $\mathrm{PTB}$, with a pooled OR of 2.84 (95\% CI 1.95-4.14; $I^{2}=23.5 \%$; Fig. 3). Meanwhile, the pooled result of cohort studies showed a similar RR of 2.37 (95\% CI 1.68-3.35; $I^{2}=0.0 \%$; Table 2).

\section{Association between HPV Infection and Clinical Pregnancy Rate of ART, Spontaneous Abortion of ART Pregnancy}

Seven cohort studies [10-12, 19-22], including 4 prospective cohort studies and 3 retrospective cohort studies, with a total of 1,390 participants, reported the impact of HPV infection on the risk of clinical pregnancy rate of ART. The pooled result of all 7 cohort studies indicated no significant association between HPV infection and clinical pregnancy rate of ART, with a pooled RR of 1.04 (95\% CI $0.64-1.70 ; I^{2}=74.3 \%$; Fig. 4). Similar results were obtained upon stratified analyses of prospective cohort studies [10$12,19]$ and retrospective cohort studies [20-22], with RRs being 1.30 (95\% CI $\left.0.61-2.80 ; I^{2}=82.5 \%\right)$ and $0.82(95 \%$ CI $0.37-1.81 ; I^{2}=69.1 \%$ ) respectively (Table 2 ). Of the 7 studies, 3 studies $[12,20,21]$ reported infection with HRHPV alone and the other 4 studies $[10,11,19,22]$ reported mixed-type infection with HR/LR-HPV, with pooled RRs of 1.66 (95\% CI $\left.0.29-9.63 ; I^{2}=90.7 \%\right)$ and 0.85 (95\% CI $0.66-1.09 ; I^{2}=20.2 \%$ ) respectively (Table 2 ). In addition, 


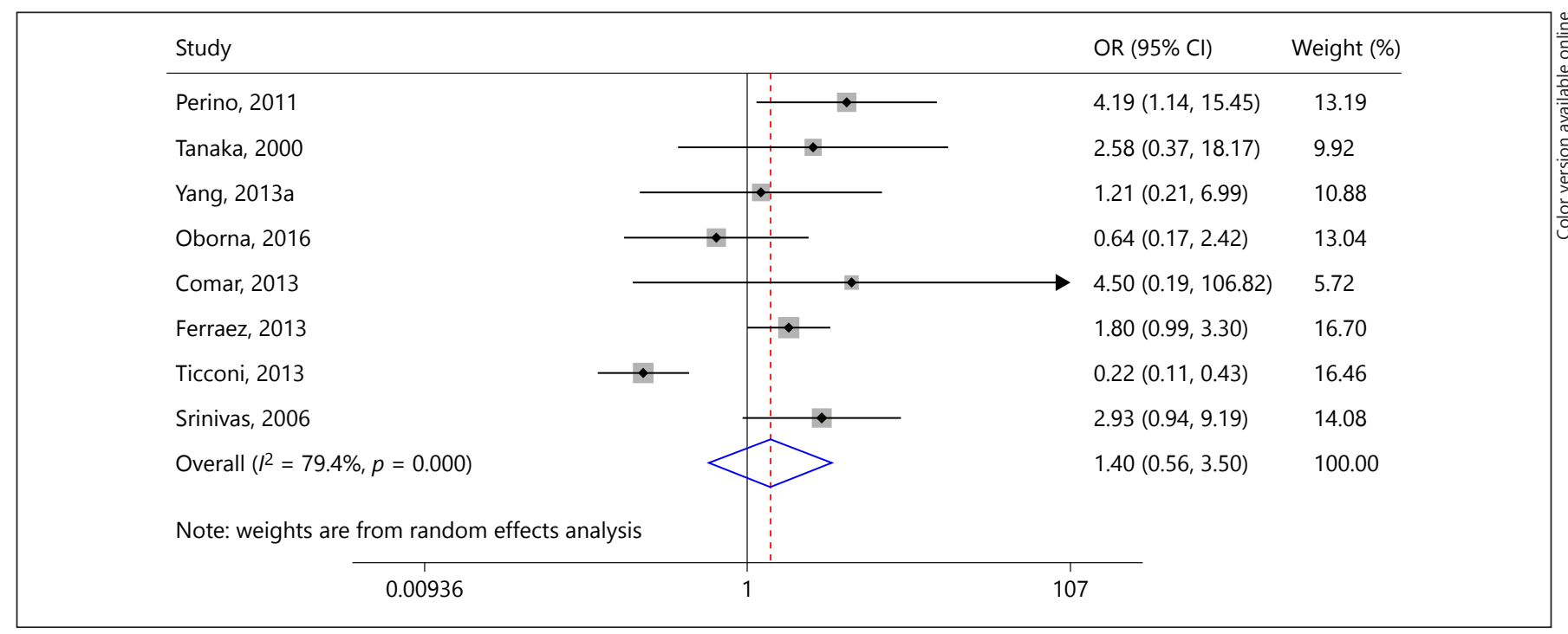

Fig. 2. Forest plot of the association between human papillomavirus infection and risk of spontaneous abortion.

Table 2. Subgroup analysis of association between HPV infection and spontaneous abortion, spontaneous preterm birth and pregnancy rate of ART

\begin{tabular}{lllll}
$\begin{array}{l}\text { Study } \\
\text { included }\end{array}$ & $\begin{array}{l}\text { Number of } \\
\text { participants }\end{array}$ & OR/RR & $95 \% \mathrm{CI}$ & $\begin{array}{l}\text { Heterogeneity } \\
\left(I^{2}\right), \%\end{array}$ \\
\hline
\end{tabular}

Spontaneous abortion

Study design

Cohort/case control study

Cohort study

Case control study

Cohort/case control and

Cross-sectional study

Genotype of HPV infection (only

cohort and case control study included)

Only HR-HPV included

Only HR/LR-HPV included

$\begin{array}{rrrrr}8 & 1,167 & 1.40 & 0.56-3.50 & 79.4 \\ 5 & 266 & 1.47 & 0.86-2.50 & 12.9 \\ 3 & 901 & 1.02 & 0.21-5.02 & 92.4 \\ 12 & 3,007 & 1.45 & 0.84-2.52 & 77.4\end{array}$

Spontaneous preterm birth

Study design

Cohort/case control study

Cohort study

Case control study

Pregnancy rate of $A R T$

Study design

Prospective/retrospective cohort study

Prospective cohort study

Retrospective cohort study

Genotype of HPV infection

Only HR-HPV included

Only HR/LR-HPV included

$\begin{array}{rrrrr}4 & 711 & 0.65 & 0.21-1.98 & 64.7 \\ 4 & 456 & 2.24 & 1.37-3.65 & 0.0 \\ & & & & \\ 4 & 1,408 & 2.84 & 1.95-4.14 & 23.5 \\ 3 & 1,348 & 2.37 & 1.68-3.35 & 0.0 \\ 1 & 60 & 6.00 & 1.17-30.73 & \text { NA }\end{array}$

HPV, human papillomavirus; HR-HPV, high risk HPV; LR-HPV, low risk HPV; NA, not available. 


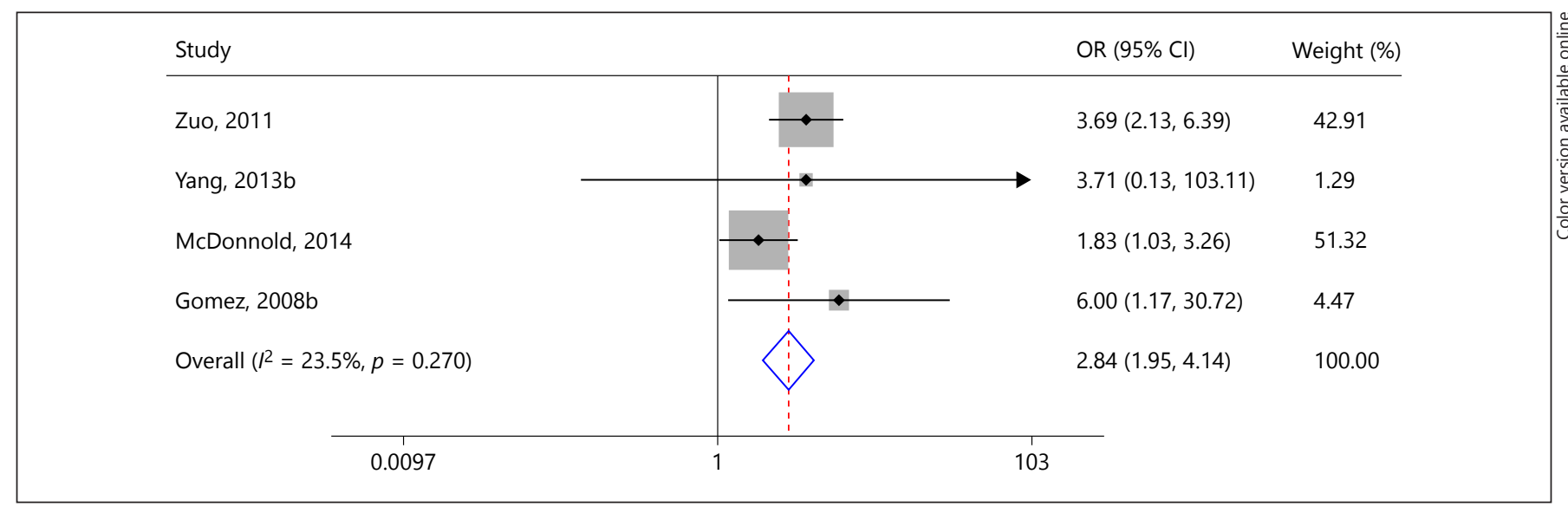

Fig. 3. Forest plot of the association between human papillomavirus infection and risk of spontaneous preterm birth.

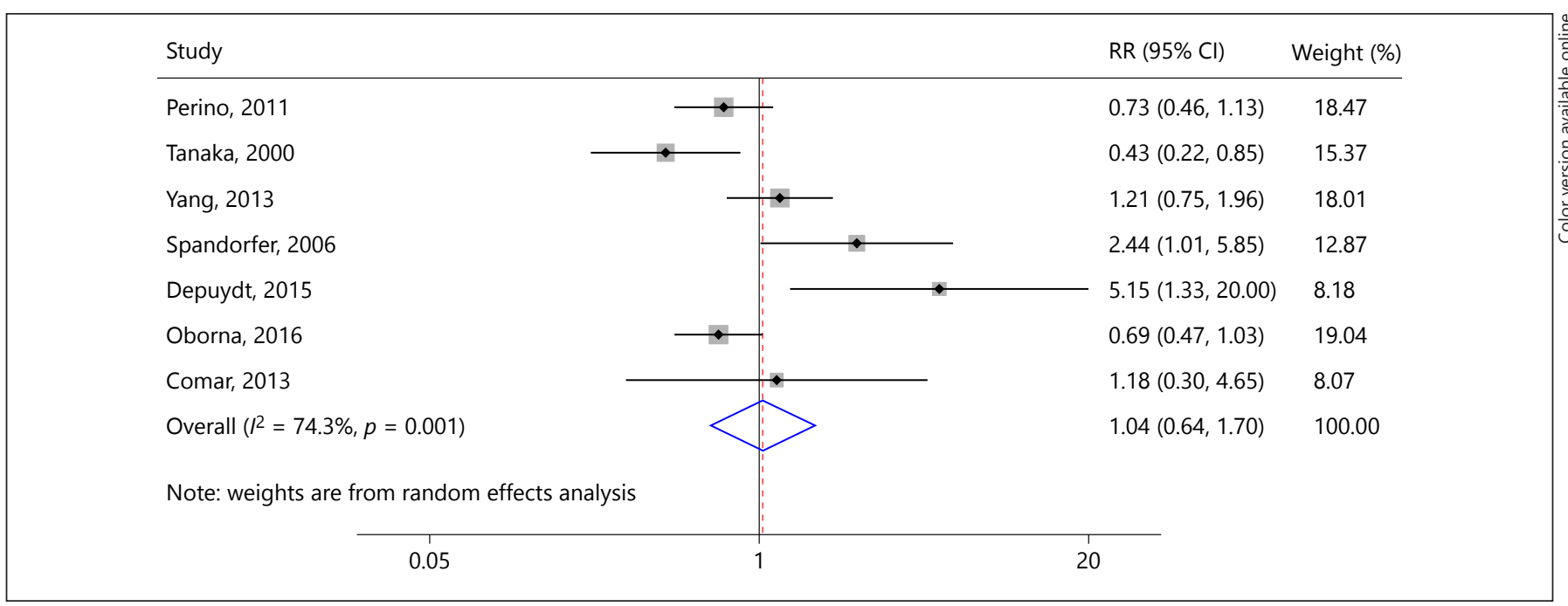

Fig. 4. Forest plot of the association between human papillomavirus infection and risk of pregnancy rate of assisted reproductive technologies.

5 studies $[12,19-22]$ reported the risk of HPV infection on spontaneous abortion of ART pregnancy, with the pooled RR being 1.47 (95\% CI 0.86-2.50; $I^{2}=12.9 \%$ ).

\section{Heterogeneity Analysis}

The inter-study heterogeneity in the analysis of impact of HPV infection on spontaneous abortion and pregnancy rate of ART was explored by meta-regression analysis. The following four variables were included in the metaregression analysis: (1) study design (cohort study vs. case-control study); (2) HPV genotype (HR-HPV infection alone vs. HR/LR-HPV infection); (3) HPV detection method (Hybrid Capture 2 Modular System vs. others); and (4) number of participants $(\geq 100 \mathrm{vs.}<100)$. The HPV genotype $(p=0.043)$ and HPV detection method ( $p=$ 0.016 ) had significant influence on heterogeneity of association between HPV infection and spontaneous abortion. HPV detection method ( $p=0.035)$ had significant influence on heterogeneity of association between HPV infection and pregnancy rate of ART.

\section{Sensitivity Analysis}

We performed a sensitivity analysis to evaluate whether any single study dominated the results of the meta-analyses by sequentially removing individual eligible studies. We observed that no individual study altered the overall 
significance of the ORs/RRs in the analyses of the impact of HPV infection on spontaneous abortion, sPTB, and outcome of ART. Additionally, we added 4 cross-sectional studies $[25,26,28,29]$ to the analysis of association between HPV infection and spontaneous abortion. The summary result of cohort, case-control, and cross-sectional studies was similar to the previous summary result of cohort/case-control studies (1.45 [95\% CI 0.84-2.52; $I^{2}=$ $77.4 \%$ ] vs. 1.40 [95\% CI $\left.0.56-3.50 ; I^{2}=79.4 \%\right]$; Table 2 ).

\section{Published Bias}

The funnel plots for publication bias were symmetrical and Egger's test indicated no significant asymmetry was found in any of the analyses.

\section{Discussion}

To the best of our knowledge, this is the first systematic literature review and meta-analysis on the published evidence of the impact of HPV infection on spontaneous abortion, clinical pregnancy rate of female undergoing ART, and spontaneous abortion of ART pregnancy. Our results indicated that there was no significant association between HPV infection and spontaneous abortion (OR 1.40; 95\% CI 0.56-3.50), clinical pregnancy rate of ART (RR 1.04; 95\% CI 0.64-1.70), and spontaneous abortion of ART pregnancy (RR 1.47; 95\% CI 0.86-2.50). While, indiscriminate genotype HPV infection increased the ratio of spontaneous abortion with pooled OR of 2.24 (95\% CI 1.37-3.65), HPV infection could increase the risk of sPTB with pooled OR of 2.84 (95\% CI 1.95-4.14).

Spontaneous abortion has been reportedly associated with several factors such as intrauterine infection, uterine malformations, fetal and placental anomalies, genetic factors, and environmental exposures [32,33]. Srinivas et al. [30] reported that the infection rates for all cytomegalovirus, HPV, or adeno-associated virus-2 groups and for any bacteria of Group B Streptococcus, Ureoplasma urealyticum, or Mycoplasma hominis were 44 and $19 \%$, respectively, in the control group, whereas the infection rates for any viruses and for any bacteria were 79 and $27 \%$, respectively, in the spontaneous case group. Although the authors did not report the co-infection rate for HPV and other viruses or bacteria, the results indicated that co-infection for HPV and other intrauterine infection were not rare in pregnant women. In the included studies for analysis of spontaneous abortion in our study, only Perino et al. [19] stated that all study participants tested negative for HIV, Chlamydia trachoma- tis, Neisseria gonorrhoeae, herpes simplex, or Treponema pallidum. Additionally, the authors reported a significant association between HPV infection and spontaneous abortion (OR 4.19, 95\% CI 1.14-15.45). As most original results included in our study lacked adjustment, some other intrauterine infection, genetic factors, and environmental exposures may have had confounding effects on the association between HPV infection and spontaneous abortion. Additional prospective studies that exclude the co-infection of HPV and other intrauterine infection in participants could be more accurate to uncover the association between HPV infection and adverse pregnancy outcomes.

HPV infection is common in the general population, including normal pregnant women. In normal full-term pregnancy, prevalence of HPV was reported to vary between 2.2 and $75 \%$ in the cervical tissue, with a summary estimate of $17.5 \%$ [7]. In placental and aborted tissues, the infection rate was slightly lower, with a summary estimate of $8.3 \%$ (95\% CI 7.6-9.1). Correspondingly, the prevalence of HPV was significantly higher in the cervix and placental and aborted tissue of women who underwent spontaneous abortions [7]. Although the prevalence of HPV was significantly different in normal pregnancies and the spontaneous abortion group, our results based on 5 cohort studies and 3 case-control studies indicated that there was no significant association between HPV infection and spontaneous abortion (OR 1.40; 95\% CI 0.563.50). When the included studies reported HR-HPV infection alone, the pooled OR was 0.65 (95\% CI 0.21-1.98). However, the pooled OR of 4 cohort/case-control studies $[19,22,24,30]$ reported that the HR/LR-HPV infection indicated that HPV infection was a risk factor for spontaneous abortion with an OR of 2.24 (95\% CI 1.37-3.65). As the high- or LR-HPV genotype was classified according to the influence of different neoplasms, the risk of these HPV genotypes for adverse pregnancy outcomes had not been well investigated. Those studies that only detected HRHPV infection may reflect a decreased rate of HPV infection in the spontaneous abortion group. Further research is still required to clarify whether certain HR-HPV or LRHPV genotypes increase miscarriage risk.

A previous meta-analysis reported that HPV infection was a risk factor for preterm birth, with an OR of 2.12 (95\% CI 1.51-2.98) [34]. We observed that 2 of its included studies $[31,35]$ recruited a component of HPV noninfection but subjects with abnormal cervical cytology in the HPV infection group; this might influence the real association between HPV infection and preterm birth. After eliminating the disputable studies, our results indi- 
cated that HR-HPV infection remained a risk for $\mathrm{sPTB}$ with a pooled OR of 2.84 (95\% CI 1.95-4.14).

Sexually transmitted infections such as Chlamydia trachomatis, Neisseria gonorrhoeae, and HPV may affect fertility or reproductive outcomes [36,37]. Our meta-analysis results showed that there was no significant association between HPV infection and pregnancy rate of ART, with a pooled RR of 1.04 (95\% CI 0.64-1.70). Meanwhile, the pooled results of 4 prospective cohort studies [10-12, 19] suggested that HPV infection may increase the risk of spontaneous abortion of ART pregnancy, but the RR was not statistically significant (RR 1.30; 95\% CI 0.61-2.80). We observed that of 7 cohort studies [10-12, 19-22] involving a total of 1,390 participants that were included in the summary analysis, only 210 subjects were positive for HPV infection. Therefore, the results of association between HPV infection and ART outcome in this study should be interpreted with caution. In a prospective study [19], the authors detected HPV infection in both women and their male partners among couples who underwent ART cycles. The result showed that couples experienced an increased risk of pregnancy loss when HPV was positive in the male partner, as compared to noninfected patients ( 66.7 vs. $15 \%, p<0.01$ ); this indicated that HPV infection in the male partner could play a role that cannot be neglected in pregnancy loss among couples who are undergoing ART. Prospective cohort studies with larger sample sizes are required to clarify whether HPV infection in women and their male partners could alter the ART outcome.

It was reported that there are 2 different pathways in the natural history of HPV infections, namely, the infectious virion producing pathway and the clonal transforming pathway [38]. The infectious virion producing pathway can lead to subfertility or early abortion and it is infectious. The clonal transforming pathway can lead to cancer but is noninfectious. The 2 pathways of HPV infection can occur simultaneously at a single location (e.g., cervix) in patients; however, it is in separate cells at different physical locations within the epidermis or mucosa [39-41]. Oncogenic (HR) HPV types induce more rapid cell division arrest than LR-HPV or intermediate HRHPV types. It takes the embryo longer to die after LRHPV infection, which makes it possible to measure the spontaneous abortion [38]. When HR-HPV virions (e.g., HPV 16) infect the embryo, the cell division should arrest over 2 cell stage. HR-HPV (e.g., HPV 53) once transferred to the embryo will first replicate each time cell division occurs. Because of the less oncogenic character of LR$\mathrm{HPV}$, the embryo will not die from division arrest but only weeks later when the dividing cells (with LR-HPV inside) differentiate into syncytiotrophoblast. Accumulation of HPV virions in the trophoblasts that are responsible for maintaining placental contact with maternal tissue and through which nutrient exchange occurs prompted the hypothesis that HPV-infected trophoblasts may have altered characteristics that may lead to a compromised gestation [38]. These may explain why when including LR-HPV in our analysis, the result indicated a significant association between HPV infection and spontaneous abortion with OR of 2.24 (95\% CI 1.37-3.65), while when only HR-HPV was included, no significant association was found (OR 0.65, 95\% CI 0.21-1.98). In order to assess the impact of the HPV infection for fertility, it is necessary to identify the origin of the detected HPV DNA (the infectious virion-producing pathway or the clonal-transforming pathway). Unfortunately, the HPV DNA tests used in the 18 studies included in our meta-analysis could not identify the origin of the detected HPV DNA. Further researches, which separate analysis the HR-HPV or LR-HPV and identify the origin of HPV, are still required to investigate the impact of HPV infection on adverse pregnancy outcomes.

Since 2006, 3 vaccines (bivalent, quadrivalent, and 9-valent) against HPV have been available. HPV vaccination programs have been implemented in many countries worldwide to prevent the high mortality from cervical cancer and to lower the incidence of dysplasia and genital warts [42]. Considering the high prevalence of HPV infection in spontaneous abortion, preterm birth, and male and female infertility, it remains uncertain whether such vaccination programs will improve the pregnancy outcome and infertility, and further investigation is required.

Some limitations of this meta-analysis should be addressed. First, there was significant heterogeneity among the included studies. Different participant inclusion and exclusion criteria, HPV-detection methods, and participants' ethnicities could have contributed to the heterogeneity. Although we performed subgroup analysis and meta-regression, the results did not identify all the heterogeneity among studies. Second, the genotypes of HPV detection were varied in the included studies; some of the studies only detected types 16 and 18, or 13 HR-HPV genotypes, whereas other studies reported both HR-HPV and LR-HPV infection. Because the influences of HRHPV and LR-HPV infection on adverse pregnancy outcomes and infertility have not been well expounded, the varied HPV genotypes in our included studies could have had some as yet unidentified influence on the pooled results. Third, HPV infection may play diverse risk in different stages of pregnancy. We did not distinguish the 
risk of HPV infection for spontaneous abortion in first, second, or third trimesters, since the time of abortion was reported inadequately in original studies. Fourth, since most studies included in our analysis did not report other adverse pregnancy outcomes risk factors, such as age of mother, ethnic origin, psychological state of the mother, smoking, alcohol consumption and a number of infections (malaria, brucellosis, human immunodeficiency virus, influenza virus and so on), we could only calculate unadjusted pooled RRs/ORs. Hence, the unadjusted pooled RRs/ORs results in our study should be interpreted with caution.

\section{Conclusion}

Indiscriminate HPV genotype infection can increase the risk of spontaneous abortion and HR-HPV infection was a risk factor for SPTB. However, there was not enough evidence to indicate the association between HPV infec- tion and pregnancy rate of ART, and spontaneous abortion of ART pregnancy. Different genotypes of HPV infection (HR-HPV or LR-HPV) may play varied roles in adverse pregnancy outcomes; hence, further epidemiologic research is required to clarify whether HPV infections indeed increase adverse pregnancy outcomes.

\section{Ethical Standards}

This article does not contain any studies with human participants.

\section{Disclosure Statement}

The authors have no conflicts of interest to disclose.

\section{Funding Sources}

The authors have no funding sources to disclose.

\section{References}

$>_{1}$ Bosch FX, Broker TR, Forman D, Moscicki AB, Gillison ML, Doorbar J, Stern PL, Stanley M, Arbyn M, Poljak M, Cuzick J, Castle PE, Schiller JT, Markowitz LE, Fisher WA, Canfell K, Denny LA, Franco EL, Steben M, Kane MA, Schiffman M, Meijer CJ, Sankaranarayanan R, Castellsague X, Kim JJ, Brotons M, Alemany L, Albero G, Diaz M, de Sanjose S; authors of ICO Monograph Comprehensive Control of HPV Infections and Related Diseases Vaccine Volume: Comprehensive control of human papillomavirus infections and related diseases. Vaccine 2013;31(suppl 7):H1-H31.

-2 Bernard HU, Burk RD, Chen Z, van Doorslaer $\mathrm{K}$, zur Hausen H, de Villiers EM: Classification of papillomaviruses (PVs) based on 189 PV types and proposal of taxonomic amendments. Virology 2010;401:70-79.

-3 Schiffman M, Clifford G, Buonaguro FM: Classification of weakly carcinogenic human papillomavirus types: addressing the limits of epidemiology at the borderline. Infect Agent Cancer 2009;4:8.

4 Damin DC, Ziegelmann PK, Damin AP: Human papillomavirus infection and colorectal cancer risk: a meta-analysis. Colorectal Dis 2013; 15:e420-e428.

5 Shaikh MH, McMillan NA, Johnson NW: HPV-associated head and neck cancers in the asia pacific: a critical literature review \& metaanalysis. Cancer Epidemiol 2015;39:923-938.

6 Conde-Ferráez L, Suarez-Allen R, ManzanoCabrera L, Gonzalez-Losa MR, Koh-Tec G,
Perez-Tuyub M, Camara-Mejia J, CarrilloMartinez J, Puerto-Solis M: Frequent infections in mexican women experiencing spontaneous pregnancy loss. Int J Infect Dis 2010; 14:e409-e410.

7 Ambuhl LM, Baandrup U, Dybkaer K, Blaakaer J, Uldbjerg N, Sorensen S: Human papillomavirus infection as a possible cause of spontaneous abortion and spontaneous preterm delivery. Infect Dis Obstet Gynecol 2016;2016:3086036.

-8 Yang Y, Jia CW, Ma YM, Zhou LY, Wang SY: Correlation between HPV sperm infection and male infertility. Asian J Androl 2013;15: 529-532.

-9 Foresta C, Pizzol D, Moretti A, Barzon L, Palu G, Garolla A: Clinical and prognostic significance of human papillomavirus DNA in the sperm or exfoliated cells of infertile patients and subjects with risk factors. Fertil Steril 2010;94:1723-1727.

10 Spandorfer SD, Bongiovanni AM, Fasioulotis S, Rosenwaks Z, Ledger WJ, Witkin SS: Prevalence of cervical human papillomavirus in women undergoing in vitro fertilization and association with outcome. Fertil Steril 2006; 86:765-767.

$\checkmark 11$ Depuydt CE, Verstraete L, Berth M, Beert J, Bogers JP, Salembier G, Vereecken AJ, Bosmans E: Human papillomavirus positivity in women undergoing intrauterine insemination has a negative effect on pregnancy rates. Gynecol Obstet Invest 2016;81:4146.
12 Oborna I, Ondryasova H, Zborilova B, Brezinova J, Vrbkova J: Does presence of human papillomavirus (HPV) infection influence the results of in vitro fertilization (IVF) treatment? Fertil Steril 2016;106:e335-e336.

13 Moher D, Liberati A, Tetzlaff J, Altman DG; PRISMA Group: Preferred reporting items for systematic reviews and meta-analyses: the prisma statement. Ann Intern Med 2009;151: 264-269, W64.

14 Stang A: Critical evaluation of the newcastleottawa scale for the assessment of the quality of nonrandomized studies in meta-analyses. Eur J Epidemiol 2010;25:603-605.

15 Wells GA Shea B, O'Connell D, Peterson J, Welch V, Losos M, et al: The Newcastle-Ottawa Scale (NOS) for Assessing the Quality if Nonrandomized Studies in Meta-Analyses. http://wwwohrica/programs/clinical_epidemiology/ oxfordhtm (cited October 19, 2009).

16 Egger M, Davey Smith G, Schneider M, Minder C: Bias in meta-analysis detected by a simple, graphical test. BMJ 1997;315:629-634.

17 Zuo Z, Goel S, Carter JE: Association of cervical cytology and HPV DNA status during pregnancy with placental abnormalities and preterm birth. Am J Clin Pathol 2011;136: 260-265.

18 Cho G, Min KJ, Hong HR, Kim S, Hong JH, Lee JK, Oh MJ, Kim H: High-risk human papillomavirus infection is associated with premature rupture of membranes. BMC Pregnancy Childbirth 2013;13:173. 
19 Perino A, Giovannelli L, Schillaci R, Ruvolo G, Fiorentino FP, Alimondi P, Cefalu E, Ammatuna P: Human papillomavirus infection in couples undergoing in vitro fertilization procedures: impact on reproductive outcomes. Fertil Steril 2011;95:1845-1848.

-20 Tanaka H, Karube A, Kodama H, Fukuda J, Tanaka T: Mass screening for human papillomavirus type 16 infection in infertile couples. J Reprod Med 2000;45:907-911.

-21 Yang R, Wang Y, Qiao J, Liu P, Geng L, Guo YL: Does human papillomavirus infection do harm to in-vitro fertilization outcomes and subsequent pregnancy outcomes? Chin Med J (Engl) 2013;126:683-687.

-22 Comar M, Monasta L, Zanotta N, Vecchi Brumatti L, Ricci G, Zauli G: Human papillomavirus infection is associated with decreased levels of GM-CSF in cervico-vaginal fluid of infected women. J Clin Virol 2013;58:479481.

23 McDonnold M, Dunn H, Hester A, Pacheco LD, Hankins GD, Saade GR, Costantine MM: High risk human papillomavirus at entry to prenatal care and risk of preeclampsia. Am J Obstet Gynecol 2014;210:138.e1-e5.

24 Conde-Ferraez L, Chan May Ade A, Carrillo-Martinez JR, Ayora-Talavera G, Gonzalez-Losa Mdel R: Human papillomavirus infection and spontaneous abortion: A casecontrol study performed in mexico. Eur J Obstet Gynecol Reprod Biol 2013;170:468473.

25 Hermonat PL, Han L, Wendel PJ, Quirk JG, Stern S, Lowery CL, Rechtin TM: Human papillomavirus is more prevalent in first trimester spontaneously aborted products of conception compared to elective specimens. Virus Genes 1997;14:13-17.

26 Skoczynski M, Gozdzicka-Jozefiak A, Kwasniewska A: Prevalence of human papillomavirus in spontaneously aborted products of conception. Acta Obstet Gynecol Scand 2011; 90:1402-1405.
27 Ticconi C, Pietropolli A, Fabbri G, Capogna MV, Perno CF, Piccione E: Recurrent miscarriage and cervical human papillomavirus infection. Am J Reprod Immunol 2013;70:343346.

28 Bennani B, Bennis S, Nejjari C, Ouafik L, Melhouf MA, El Rhazi K, Znati K, Chaara H, Bouchikhi C, Amarti Riffi A: Correlates of HPV: a cross-sectional study in women with normal cytology in north-central morocco. J Infect Dev Ctries 2012;6:543-550.

29 Joshi S, Babu JM, Jayalakshmi D, Kulkarni V, Divate U, Muwonge R, Gheit T, Tommasino M, Sankaranarayanan R, Pillai MR: Human papillomavirus infection among human immunodeficiency virus-infected women in maharashtra, india. Vaccine 2014;32:10791085 .

30 Srinivas SK, Ma Y, Sammel MD, Chou D, McGrath C, Parry S, Elovitz MA: Placental inflammation and viral infection are implicated in second trimester pregnancy loss. Am J Obstet Gynecol 2006;195:797-802.

31 Gomez LM, Ma Y, Ho C, McGrath CM, Nelson DB, Parry S: Placental infection with human papillomavirus is associated with spontaneous preterm delivery. Hum Reprod 2008; 23:709-715.

32 Donders GG, Van Bulck B, Caudron J, Londers L, Vereecken A, Spitz B: Relationship of bacterial vaginosis and mycoplasmas to the risk of spontaneous abortion. Am J Obstet Gynecol 2000;183:431-437.

33 Gravett MG, Rubens CE, Nunes TM; GAPPS Review Group: Global report on preterm birth and stillbirth (2 of 7): Discovery science. BMC Pregnancy Childbirth 2010;10(suppl 1):S2.

- 34 Huang QT, Zhong M, Gao YF, Huang LP, Huang Q, Wang W, Wang ZJ, Yu YH: Can HPV vaccine have other health benefits more than cancer prevention? A systematic review of association between cervical HPV infection and preterm birth. J Clin Virol 2014;61:321-328.
5 Hong JN, Berggren EK, Campbell SL, Smith JS, Rahangdale L: Abnormal cervical cancer screening in pregnancy and preterm delivery. Paediatr Perinat Epidemiol 2014;28:297301.

36 Souho T, Benlemlih M, Bennani B: Human papillomavirus infection and fertility alteration: a systematic review. PLoS One 2015; 10:e0126936.

37 Pereira N, Kucharczyk KM, Estes JL, Gerber RS, Lekovich JP, Elias RT, Spandorfer SD: Human papillomavirus infection, infertility, and assisted reproductive outcomes. J Pathog 2015;2015:578423.

- 38 Depuydt CE, Beert J, Bosmans E, Salembier G: Human papillomavirus (HPV) virion induced cancer and subfertility, two sides of the same coin. Facts Views Vis ObGyn 2016;8: 211-222.

39 Depuydt CE, Jonckheere J, Berth M, Salembier GM, Vereecken AJ, Bogers JJ: Serial typespecific human papillomavirus (HPV) load measurement allows differentiation between regressing cervical lesions and serial virion productive transient infections. Cancer medicine 2015;4:1294-1302.

40 Depuydt CE, Thys S, Beert J, Jonckheere J, Salembier G, Bogers JJ: Linear viral load increase of a single HPV-type in women with multiple HPV infections predicts progression to cervical cancer. Int J Cancer 2016;139: 2021-2032.

41 Verhelst S, Poppe WA, Bogers JJ, Depuydt CE: Serial measurement of type-specific human papillomavirus load enables classification of cervical intraepithelial neoplasia lesions according to occurring human papillomavirus-induced pathway. Eur J Cancer Prev 2017;26:156-164.

42 Bonde U, Joergensen JS, Mogensen O, Lamont RF: The potential role of HPV vaccination in the prevention of infectious complications of pregnancy. Expert Rev Vaccines 2014;13:1307-1316. 